\title{
Erratum to: Degradation of Lignin in Agricultural Residues by locally Isolated Fungus Neurospora discreta
}

\author{
Sirisha Pamidipati ${ }^{1}$ - Asma Ahmed ${ }^{1}$
}

Published online: 5 January 2017

(C) Springer Science+Business Media New York 2016

\section{Erratum to: Appl Biochem Biotechnol (2016)}

DOI 10.1007/s12010-016-2302-6

The original version of this article unfortunately contained a mistake. The order of Figure 4 sub-panel images were placed incorrectly; the correct version is provided below and the original article has been corrected. The figure caption remains the same as before.

The online version of the original article can be found at http://dx.doi.org/10.1007/s12010-016-2302-6.

Asma Ahmed

asma.ahmed@hyderabad.bits-pilani.ac.in

1 Department of Chemical Engineering, Birla Institute of Technology and Science Pilani Hyderabad, Campus, Jawahar Nagar, Shameerpet Mandal, Hyderabad 500078, India 

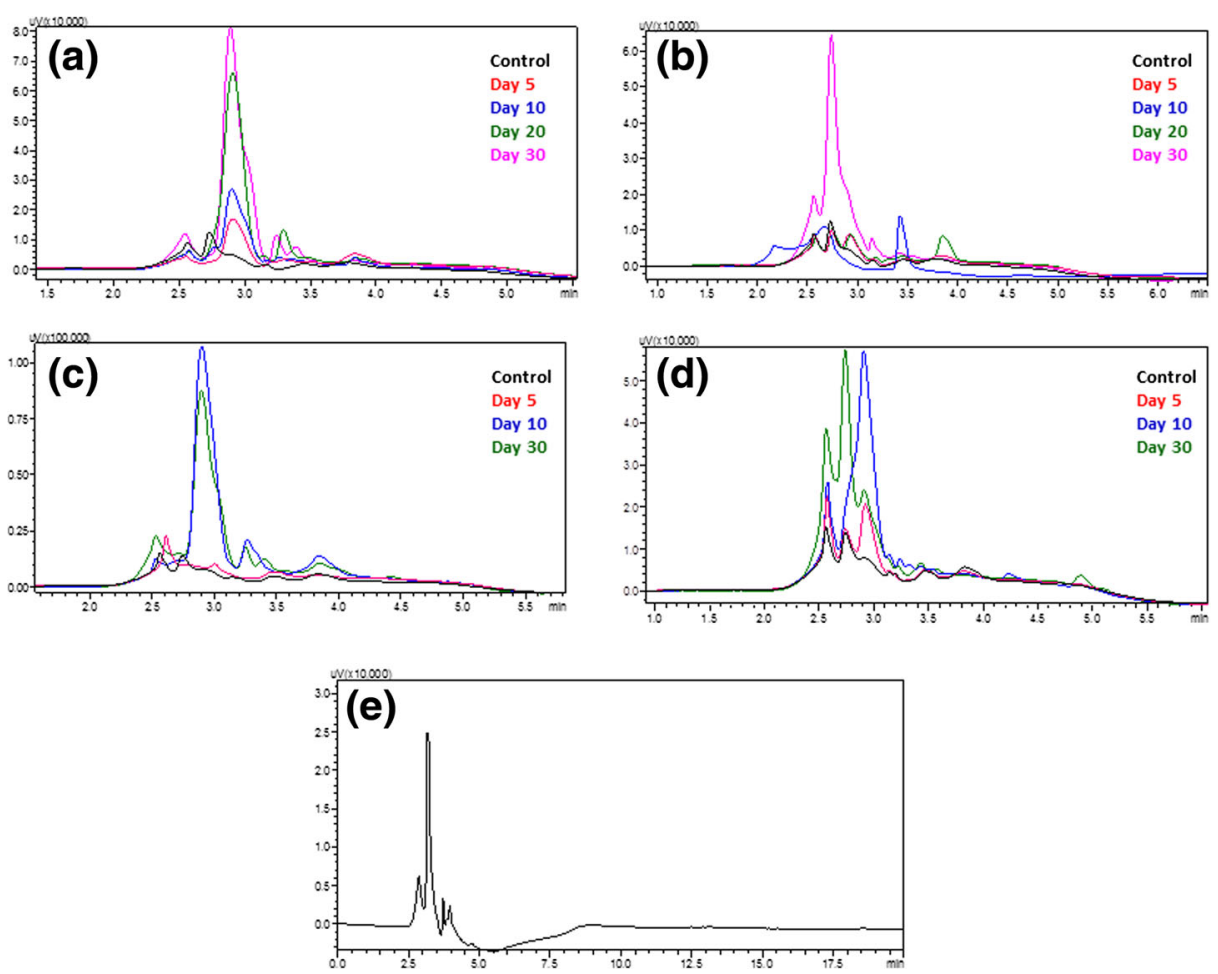

Fig. 4 Chromatograms of liquid supernatant samples of a cocopeat treated with $N$. discreta, b cocopeat treated with $P$. chrysosporium, c sugarcane bagasse treated with $N$. discreta, d sugarcane bagasse treated with $P$. chrysosporium, and e standard alkali lignin $(0.5 \mathrm{mg} / \mathrm{ml})$ 